\title{
A Novel Method For Android Version Updates To Avoid Fragmentation
}

\author{
Robin Kamboj, Bhoomi Gupta
}

\begin{abstract}
Android is the most widely used operating system worldwide, running on over seventy percent of the entire smartphone market. However, Android OS suffers from a big problem, known as fragmentation, that causes many complications both on the user side and the development side. Various solutions are currently live to eliminate these complications in the near future, but there is currently no direct solution for the same. A project was developed with the name SENSE IT ALL - DEVICE TEST to help counter and minimise these complications caused due to fragmentation on the platform. Sense It All is an Android application, built using the native Android SDK, that provides diagnostic tests to the deviceincorporated sensors, features, trending technologies, internal system, and other tools, to help a naive user, or a sophisticated user, to understand, reduce, and even eliminated the consequential complications due to fragmentation.
\end{abstract}

Index Terms - Android, Project Treble, Android One Program, Sense It All, Device Test, Fragmentation

\section{INTRODUCTION}

There has been a duopoly in the smartphone operating system market for a long time now. The operating systems Android and iOS dominate over all other smartphone operating systems. According to GlobalStats website, Android OS occupies about $74 \%$ of the worldwide market shares as a smartphone OS, iOS occupies $23 \%$, KaiOS $1 \%$, Windows $0.39 \%$, Samsung $0.32 \%$ and others $0.62 \%$ [1].

Table-1: Major Mobile Operating Systems

\begin{tabular}{|l|r|}
\hline Operating System & Distribution \\
\hline Android & $74.45 \%$ \\
\hline IOS & $22.85 \%$ \\
\hline KaiOS & $1.1 \%$ \\
\hline Windows & $0.3 \%$ \\
\hline Samsung & $0.28 \%$ \\
\hline Unknown & $0.41 \%$ \\
\hline
\end{tabular}

Revised Manuscript Received on April 19, 2019.

Robin Kamboj, B.Tech Student, Maharaja Agrasen Institute Of Technology, Rohini, New Delhi, India.

Dr. Mrs. Bhoomi Gupta, Assistant Professor, Maharaja Agrasen Institute Of Technology, Rohini, New Delhi, India.
From the above data, we observe that Android and iOS alone cover $97.30 \%$ of the smartphone OS market. Also, of these two players, Android has almost three times the shares of the second biggest competitor in the same. By these facts, it is very clear that Android is the most dominant player as a smartphone operating system, and that its popularity is unmatched by any other OS at this point.

To know more about the quality of the two major players, it is required to dive deeper into their internal numbers.

The latest version of iOS, iOS 12, was launched on 17 September, 2018. According to Apple, as of December 3, 2018 (about 2.5 months since release), more than $\mathbf{7 0 \%}$ of all devices running on iOS (that is, iPhones) were running on iOS 12. On the other hand, the latest version of Android OS (Pie) was launched on 6 August, 2018, and as of December 2018 (four months since release), less that $1 \%$ of all android devices run on the latest Android version, that is, Android Pie.

It is very surprising, and at the same time, interesting to compare these statistics of the two major players in the smartphone OS market.

Apple manages to update more than $70 \%$ of devices running on iOS to its latest version within the span of nearly just two months, while Google performs miserably, and fails to get the latest android software to even on 1 percent of devices running on the Android platform.

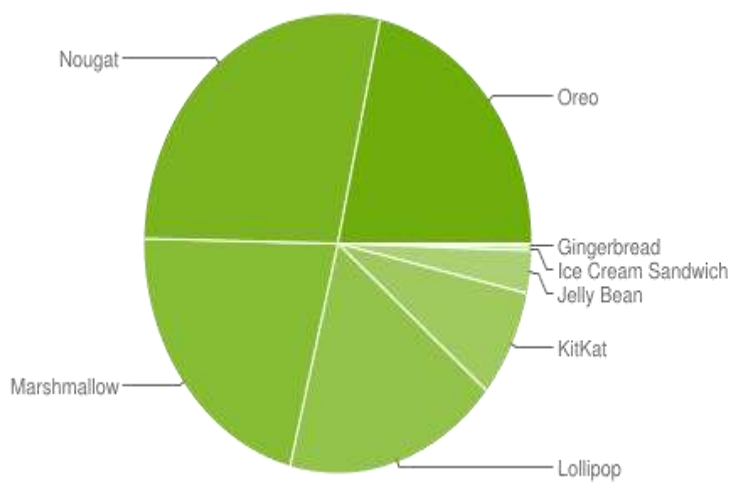

Figure-1. This image shows the Android OS version distribution in a pie chart. [2] 
It is very surprising, and at the same time, interesting to compare these statistics of the two major players in the smartphone OS market.

Apple manages to update more than $70 \%$ of devices running on iOS to its latest version within the span of nearly just two months, while Google performs miserably, and fails to get the latest android software to even on 1 percent of devices running on the Android platform.

\section{THE PROBLEM}

Table-2: Android Versions Distribution Figures

\begin{tabular}{|lr|}
\hline Version Name & Distribution \\
\hline Oreo 8.0 & $20.27 \%$ \\
\hline Marshmallow 6.0 & $17.58 \%$ \\
Oreo 8.1 & $16.78 \%$ \\
\hline Nougat 7.0 & $12.71 \%$ \\
\hline Lollipop 5.1 & $11.56 \%$ \\
\hline Nougat 7.1 & $8.86 \%$ \\
\hline
\end{tabular}

On diving deeper into the Android numbers alone, we observe that as of 28 September, 2018, about $18 \%$ of all smartphone users are running on Android version 8 (Oreo), about $29 \%$ on Android version 7 (Nougat), about $21 \%$ on version 6 (Marshmallow), about $18 \%$ on version 5 (lollipop), and about 11 percent on older versions (data by Google). [2]

In other words, the whole of 99 percent Android users are running on the software that has been outdated for at least one year: about $18 \%$ devices running on one year old technology (version 8 or Oreo), $29 \%$ on two year old technology (version 7), 21 percent on three year old technology (version 6) and so on.

This big problem of uneven distribution of software updates is known as fragmentation. Fragmentation is in Android as is no where else; being the most dominant player in the smartphone operating system market, the fragmentation problem is a pain in the neck for not just Google, but also for the millions of users who use Android OS on their smartphones as a daily driver, and then never get the latest update with time.

The fact that the fragmentation problem in Android is so severe is attributed to the fact that Android is an open source platform, and instead of rolling out their own devices like Apple, Google makes this as an open source platform available to other smartphone manufacturers to incorporate in their own physical devices.

As mentioned before, whenever Google pushes a new software update for Android, a manufacturer chooses to incorporate in their devices; but they don't do it directly they customise the original code to overlay features onto the new Android software

This is done by almost each manufacturer, because original version of android, also known as stock android, is very minimalistic, and tries to provide simple and bare minimum features that are sufficient for a good user experience. The manufacturers do this also to incorporate their platform-specific applications and portals, to promote their brand.

But when a year later, another update is made available by Google for manufacturers, the following points refrain manufacturers to update their devices to the latest update:

\section{Vendor Implementation:}

To create a smartphone body, a manufacturer usually assembles parts from different vendors; like screen from Samsung, processor chip from Qual Comm, and so on. These vendors write platform (android) specific code, to make these parts compatible with Android software. So, when a new software version comes up, a manufacturer has to make sure that all these vendors update their internal code to conform to the new Android software version.

\section{Custom Skin:}

As mentioned before, a manufacturer customises the open source Android software code to add new features. Because of extra code and time involved in executing this process, whenever a new software version comes up, these sets of features have to be rewritten based on the updated software code. [11]

\section{Before Treble}

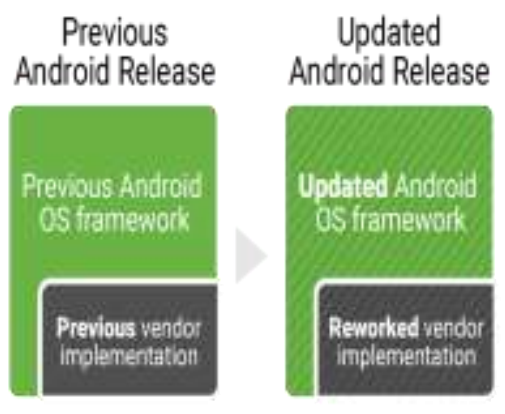

\section{Figure-2. Figure shows Android update process for} pre-Oreo devices [6]

Both these factors contribute largely to the delay in Android software updates to their devices, if at all intended.

So far, it is established that fragmentation is a big problem in Android operating system. Google is well aware of this problem, and is taking some actions to counter the same, and this part will be discussed later.

However, the results of any action are not expected to rectify this problem anytime soon.

The consequences of fragmentation in Android is most affected on two types of users:

\section{Issues for application developers.}

Due to fragmentation in Android, there are various software versions, like Oreo, Nougat, Marshmallow and Lollipop, that significantly contribute to the number of 
Android users. With each software update, new features are added to the previous version, some inefficient features are removed (deprecated), and many underlying implementations of previously existing features is changed. Due to this, developers have an easy but impractical choice to only target the latest Android version (which, as already discussed, is less than 1 percent of entire Android user base), or they have a difficult but definitive choice to cover all of these exceptional cases in their application's code. They have to write the application's code such that all previous versions should be able to support the application that they are building. Unfortunately, doing this is very tedious, and even best of the best applications on the Google Play Store are easily susceptible to plentiful bugs and crashes. [10]

Moreover, since Android is an open source platform, whose code can be borrowed by a third party manufacturer to put into their physical devices, Android software runs on thousands of devices of different shapes and sizes. An application has to be built so that the user experience is similar across all of the devices in the world.

In case of iOS, because almost all of its users are easily converted onto the latest iOS version, application developers generally only have to target the latest iOS version's code, and not any older versions. This is a relatively simple task, and iOS developers are easily able to create robust and reliable applications. As a result, iOS users are less likely to experience any crashes or bugs, and as a consequence, have better user experience.

\section{Issues for Android users:}

Due to the fact that an application developer may not be able to direct an app to conform to all Android versions and to support all screen sizes and ratios, there might be anomalies like bugs, faults and crashes due to the application. In addition, because a manufacturer, usually, adds custom skin over the original Android software code, their compatibility with all rumoured 2.6 million apps on the Google Play Store (as of December, 2018) is largely affected negatively. As a result, when an application is not working as expected in case of the user experiencing a bug, error, crash or a situation where one of the app's feature is not functioning as expected, the naive user does not know whether the issue that they are experiencing is due to the operating system or the application. In such a situation, there can be two common scenarios:

1. A user assumes that the fault is entirely of the application. In such a case, the frustrated and angry user

typically either uninstalls the app, or goes to the Google Play Store to rate the app experience negatively, or both.
2. A user worries if their phone is malfunctioning. In this case, the user is clueless as to what to do in order to verify if their device is running properly or not.

In May, 2017, Google announced that there are over 2 billion monthly active devices running on Android OS. Hence, because of the previously mentioned fact that over 99\% of devices running on Android are running on at least one year old OS version, almost all of these two billion people suffer from the problems mentioned above. Moreover, for obvious reasons, since application developers are only a tiniest of the part of entire Android user base, only naive users, or the general public, is largely affected by these problems.

The problems mentioned above lead to the following consequences that a naive user has to suffer with:

1. A user usually spends a lot of time in researching for a new device to buy. Once a user experiences negative events in their device, they get slightly demotivated on their purchase.

2. If the negative events being experienced in the device occur in high frequencies, the user is annoyed as to what is happening to the device, and why that is happening, or is confused if it is the device's fault, or the app, that causes the anomaly.

3. Also, the user has no definite way to test the feature that is in question in their mind.

A lot of efforts have been put in by independent software engineers and developers to eliminate the above mentioned problems, but no independent solution has so far been able to handle the situation successfully yet.

\section{SOLUTIONS TO THE PROBLEM}

Various device test applications have been created to help users detect any faults with the normal functioning of their devices, to give them a heads up that their device is running as expected, But none of the applications cover all the sensors and features that are present in a device, let alone the advanced features that get added with the advent of new technologies with time, like virtual reality, augmented reality, barcode scanning, face recognition, text scanning, landmark detection, label generator, etc.

In addition, since applications like this are only required to be accessed once in a long while (when a device feature is in question), they usually either sit passively in a device, or are uninstalled by a user, because utility of an app and the frequency of use of an app are the main deciding factors of usefulness to a user. As a result, applications built exclusively on providing device tests fail to be of great importance to the intended users. 
Test your device.

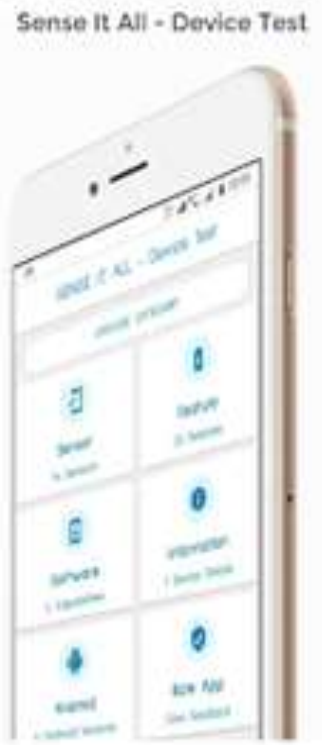

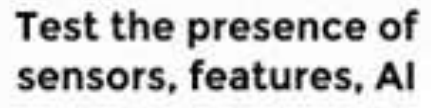

and other components

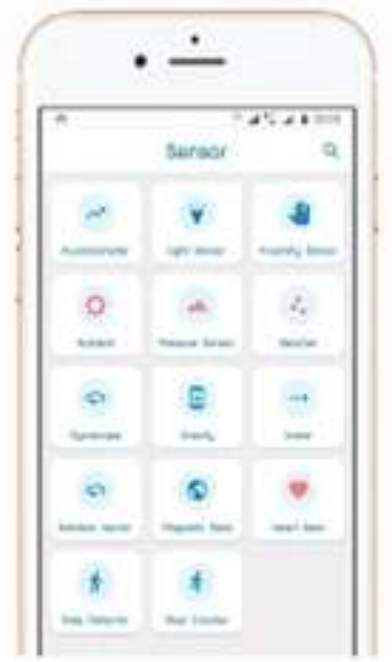

\section{Sophisticated Tests for enthusiasts}

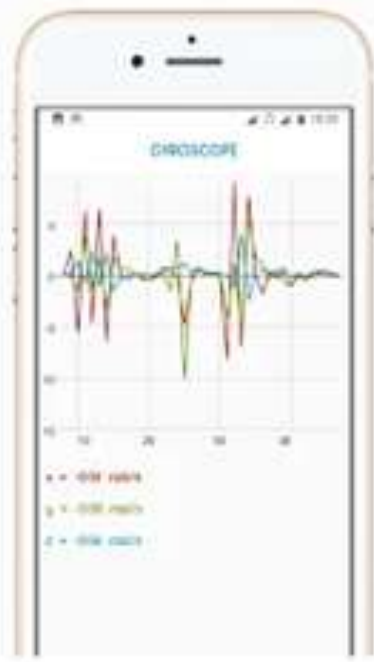

Integrat for each $\mathrm{C}$ I

ever 40 device

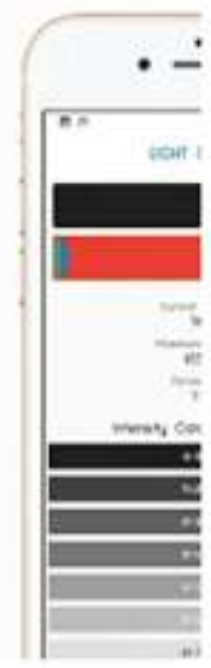

Figure 3. Google Play Store listing screenshots of the "Sense It All - Device Test" application [5]

To counter the problem, an application named "Sense It All - Device Test" was published on the Google Play Store, offered by Simplified Soul developer identity, and released on April 4, 2017. Unlike simple device test applications, Sense It All is a universal device diagnostics application that covers a wide range of traditional, core and modern tests including hardware sensors, system features, software capabilities, memory analysis and information, and modern technologies tests. To support daily user interaction, the application also features everyday tools in order to assist users to ease out their day-to-day activities related to their smartphone devices. Thus, collectively, the aim of the application is to provide users with everyday tools and also to allow them to test and explore all sensors, features, details, etc. The application is currently live on the Google Play Store with the same name. [8]

In addition, the application has been constantly evolving based on the user reviews, user feedback, etc, and also for reasons to bring down the number of bugs and crashes to a minimum level, improving app performance, enhancing user experience, and so on. So far, the application has been updated over 45 times on the Play Store. The application has organically gained popularity on the Play Store, gaining an average rating of 4.4 by its users, and obtaining over 25 thousand downloads since launch, that is, without any paid promotion or campaign.

Apart from independent developers and organisations, even Google is working on finding a solution to the fragmentation problem on the Android platform. But, unlike independent developers who have so far been working to counter the problems caused by fragmentation, Google is working on eliminating the problem completely. Google has adopted two main mechanisms that would enable smartphones to get regular and stable updates to smartphone devices:
1. Android One Program
2. Project Treble

Because of Android being an open source platform, any manufacturer can take this open source software code to put it in their devices for use by public. Because of the code being open source, a manufacturer typically adds features on top of this code, or adds a custom skin to make the software look better.

Android One program was launched by Google on August 10, 2015, to help manufacturers give their users the stock-android experience. In case of devices running on the Android One program, a manufacturer puts the original software code as it is in their devices: with no insertions of any additional features, or an addition of custom skin to make the OS look better. In addition, the Android Now program offers two major key features:

1. The device running on an Android One program will get guaranteed two years of monthly security updates.

2. The device running on the Android One program will get guaranteed updates for at least two major Android versions.

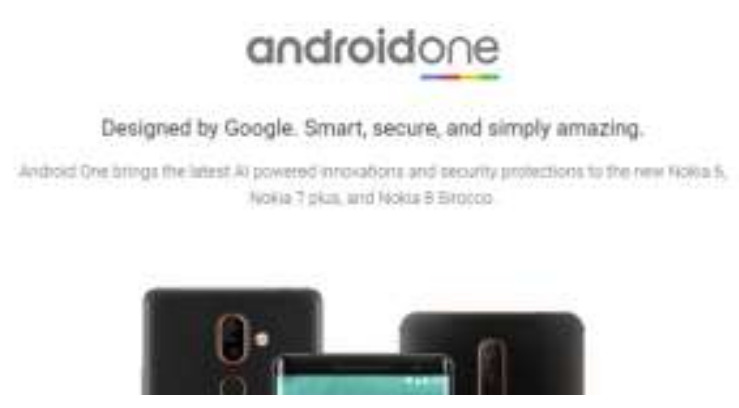

Figure-4. Android One Program launched by Google. [4] 
Project Treble was launched by Google on August 21, 2017. With project treble, starting with Android version 8, Google has separated the OS framework and low-level software.

Because of this, manufacturers will no longer have to update their low-level software code (or ask device-parts vendors to update their low-level codes) in order to push a new update onto the devices. Hence, rolling out version updates will be relatively way easier using project treble than ever before. [7]

\section{ANDROID UPDATES BEFORE TREBLE}

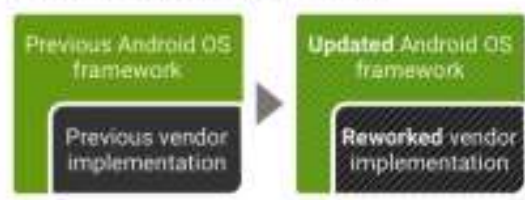

ANDROID UPDATES WITH TREBLE

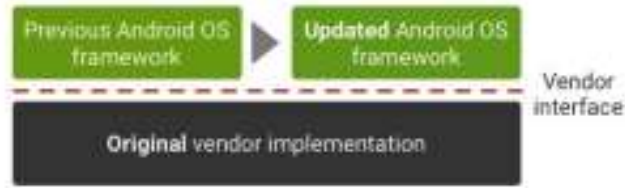

Figure-5. Android version updation process after Oreo [3]

\section{CONCLUSION}

The guaranteed updates provided by manufacturers for the devices enrolled in the Android One program will help reduce the extent of the fragmentation problems caused in the Android platform, that transitively reduces the issues caused due to it to the public.

Both of Google's solutions: Android One Program and Project Treble, have big loopholes that prevent them in making a significant contribution to resolve the Android fragmentation problem. For example, in project treble, despite the fact that it will be very easy to push software updates onto the devices, but still, it is upto the manufacturer to decide whether or not to allow an update to be rolled out onto the old devices.

In case of Android One, it is guaranteed that a device will get two major Android version updates, but it is not specified anywhere when these major updates will be pushed; a typical trend has been months of delays since the launch date of a new version.

Because of negative bypasses like these, it is highly unlikely that any of the Google's solutions will make any good impact on the problem. Sense It All - Device Test currently seems to be the best source to counter, or at least, relieve the impact of this large-scale problem onto the consumers.

\section{REFERENCES}

1 Mobile OS Market Share. Retrieved from http://gs.statcounter.com/os-marketshare/mobile/worldwide
2 Android Versions Distribution.Retrieved from https://developer.android.com/about/dashboards/

3 Android Authority. Retrieved from https://www.androidauthority.com/project-treble-818225/

4 [4] Android One. Retrieved from https://www.android.com/one

5 Sense It All, Play Store. Retrieved from https://play.google.com/store/apps/details?id=com.appbu sters.robinkamboj.senseitall

6 Android Update Process for Pre-Oreo Devices. Retrieved from https://9to5google.com/2017/05/12/google-projecttreble-android-updates/

7 Project Treble. Retrieved from https://source.android.com/devices/architecture

8 Google Play Store.Retrieved from https://developer.android.com/distribute/google-play

9 Android Fragmentation.Retrieved from https://www.techopedia.com/definition/3899/androidfragmentation

10 Android SDKRetrieved from https://developer.android.com

11 Android Skins. Retrieved from https://www.cnet.com/news/android-interface-guide/ 